This item was submitted to Loughborough's Research Repository by the author.

Items in Figshare are protected by copyright, with all rights reserved, unless otherwise indicated.

\title{
Dispersion management for solitons in a Korteweg-de Vries system
}

PLEASE CITE THE PUBLISHED VERSION

\section{LICENCE}

CC BY-NC-ND 4.0

\section{REPOSITORY RECORD}

Clarke, Simon R., Boris A. Malomed, and Roger H.J. Grimshaw. 2019. "Dispersion Management for Solitons in a Korteweg-de Vries System". figshare. https://hdl.handle.net/2134/684. 


\title{
"Dispersion management" for solitons in a Korteweg-de Vries system
}

\author{
Simon Clarke ${ }^{1}$, Boris A. Malomed ${ }^{2}$ and Roger Grimshaw ${ }^{3}$ \\ ${ }^{1}$ Department of Mathematics and Statistics, Monash University \\ Vic. 3800, Australia \\ ${ }^{2}$ Department of Interdisciplinary Studies, Faculty of Engineering, \\ Tel Aviv University, Tel Aviv, Israel \\ ${ }^{3}$ Department of Mathematical Sciences, Loughborough University, \\ Loughborough, Leicestershire LE11 3TU, UK
}

April 3, 2001

\begin{abstract}
The variable-coefficient Korteweg-de Vries is used to present a basic model, which has the form of a Korteweg-de Vries equation with a periodically varying third-order dispersion coefficient, that can take both positive and negative values. More generally, this model may be extended to include fifth-order dispersion. Such models describe, for instance, a periodically modulated waveguide for long gravity-capillary waves. We develop an analytical approximation for solitary waves in the weakly nonlinear case, from which it is possible to obtain a reduction to a relatively simple integral equation, which is readily solved numerically. Then, we describe some systematic direct simulations of the full equation, which use the soliton shape produced by the integral equation as an initial condition. These simulations reveal regions of stable and unstable pulsating solitary waves in the corresponding parametric space. Finally, we consider the effects of fifth-order dispersion.
\end{abstract}

\section{Introduction and model formulation}

Classical models which give rise to solitons, such as the Korteweg-de Vries $(\mathrm{KdV})$ or nonlinear Schrödinger (NLS) equations, describe uniform nonlinear waveguides. For example, the NLS equation is a basic model to describe light propagation in optical fibers and other guiding structures [1], while a wellknown application of the $\mathrm{KdV}$ equation is the description of internal waves in stratified fluids, propagating in waveguides which exist naturally in the ocean, or can be created in a laboratory $[2,3]$. In both these models, a (bright) soliton exists under a certain condition: in the NLS equation, the dispersion must be anomalous [1], while in the $\mathrm{KdV}$ equation, the soliton must have a definite polarity.

The necessity to improve stability, bit rate, and other operation characteristics for soliton streams in optical fibers, which are expected to be a basis for 
a new generation of optical telecommunications, have recently attracted a great deal of attention to the technique of "dispersion management" (DM), i.e., transmission of solitons in an optical fiber which consists of periodically alternating sections with opposite signs of the dispersion, so that the average dispersion of the long communication link is nearly equal to zero [4, 5]. The corresponding NLS equation with a variable dispersion coefficient is no longer integrable by means of the inverse scattering transform, and no exact soliton solution is known for it. Nevertheless, very accurate numerical simulations show that, to an extremely high accuracy, this equation gives rise to solitons with an approximately Gaussian (rather than the classical sech), pulsating shape [5, 6]. The existence of dispersion-managed solitons of this type is also strongly supported by analytical results produced by the variational approximation developed for the DM model in different forms [7, 8], as well as by analysis of the model transformed into an integral equation [9].

A remarkable property of DM solitons is that they may exist, in a stable form, even when the average dispersion, which is not necessarily exactly zero, but may be much smaller than the local dispersion in the alternating fiber segments, is normal [10,8] (recall that the classical NLS solitons cannot exist in the case of the normal dispersion). It is necessary to mention that, prior to the appearance of the DM models with the above-mentioned piece-wise constant form of the dispersion profile, the NLS equation with a sign-changing harmonically (sinusoidally) modulated dispersion term had already been studied in detail, with a conclusion, based on both the variational approximation and direct simulations, that stable pulsating solitons exist also in that smoothly modulated model [11].

In fact, the NLS equation with the local dispersion subject to strong periodic modulation belongs to a class of periodic heterogeneous systems, in which stable pulsating solitons with nontrivial properties may be expected (in this work, we use the term "soliton" without assuming integrability of the corresponding model). In nonlinear optics, other systems belonging to this class are tandem waveguides for optical solitons supported by quadratic $\left(\chi^{(2)}\right)$ nonlinearity, based on alternation of $\chi^{(2)}$ and linear segments [12], split-step fiber links, in which linear segments alternate with those dominated by the $\operatorname{Kerr}\left(\chi^{(3)}\right)$ nonlinearity [13], layered bulk media, with the $\chi^{(3)}$ coefficient varying between the layers so that an optical beam propagating across the layers has its power oscillating around a critical value leading to the wave collapse [14], and alternate nonlinear waveguides, composed of periodically alternating waveguiding and antiwaveguiding segments [15]. A remarkable feature, common to all these systems, despite their very different physical nature, is the strong robustness of the propagation modes, and the absence of any apparent instability, even when this might naively have been expected.

The identification of this class of models, essentially based on the NLS equation, makes it natural to ask whether other classical soliton-generating equations, if subjected to a periodic modulation of the dispersion coefficient, can give rise to the propagation of pulsating robust solitary wave. The first candidate to be investigated is the Korteweg-de Vries (KdV) equation. In fact, the $\mathrm{KdV}$ equation with variable coefficients is a traditional object for the application of the perturbation theory for solitons [16]. However, the case of periodic modulation, and the consequent possibility of the existence of a quasi-stable pulsating soliton in this case has not yet been studied. 
We start with consideration of the propagation of weakly nonlinear long waves in a periodically inhomogeneous waveguide, based on the variable-coefficient KdV equation:

$$
u_{t}+c(\epsilon x) u_{x}+\epsilon\left(r(\epsilon x) u u_{x}+s(\epsilon x) u_{x x x}\right)=0\left(\epsilon^{2}\right),
$$

where $\epsilon \ll 1$ is a basic small parameter. Equations of this general form are commonly used to describe the propagation of solitary waves in inhomogeneous media, for instance, internal waves in stratified fluids inhomogeneous in the longitudinal direction, which is a generic case for internal waves in the ocean [3]. Provided that the local nonlinear coefficient, $r$, and phase velocity, $c$, are nonvanishing functions of $\epsilon x$, one can introduce a propagation coordinate $\chi$ and a temporal variable $\theta$ as follows:

$$
\chi=-\int \frac{\epsilon r}{6 c^{2}} d x, \quad \theta=t-\int \frac{d x}{c} .
$$

Then Eq. (1) reduces, in the same approximation at which it is valid, to

$$
u_{\chi}+6 u u_{\theta}+D(\chi) u_{\theta \theta \theta}=0
$$

where the local dispersion coefficient in the modulated system is

$$
D(\chi)=6 s /\left(r c^{2}\right)
$$

Two integral quantities, which are frequently called mass and momentum,

$$
M=\int_{-\infty}^{+\infty} u(\theta) d \theta, P=\int_{-\infty}^{+\infty} u^{2}(\theta) d \theta,
$$

are exact dynamical invariants of Eq. (3), i.e., $d M / d \chi=d P / d \chi=0$. Note that in this context $P$ is more accurately described as the wave action flux.

Our objective is to consider the case when $D(\chi)$ is a periodic function, and in particular, when it periodically changes its sign. For instance, as is shown in the Appendix, Eq. (3) describes the propagation of gravity-capillary waves over a periodically-varying-bottom topography near the critical Bond number. It is also a possible model equation for the propagation of internal waves and Rossby waves against the background of a slowly varying shear flow [2]. In the latter case, wave modes in critical layers, where the long-wave speed is within the range of the shear velocity, may have both positive and negative third-order dispersion coefficients. Generally speaking, in all these applications, one should also take into regard the fifth-order dispersion; nevertheless, it will initially be assumed here that the higher-order dispersion is negligible.

We assume that the variable dispersion coefficient $D(\chi)$ in Eq. (3) can be represented in the following, quite general, form:

$$
D=S D_{0}(\chi / T)+D_{1}(\chi)
$$

where $D_{0}$ is a periodic function with period 1 (i.e., the actual period of the first term in Eq. (6) is $T$ ), mean value exactly equal to zero, and amplitude 1. Following the analogy with the DM models in nonlinear optics, we will then refer to the parameter $S$ as the map strength. The function $D_{1}$ then 
represents the local average dispersion, which may also be subjected to a longrange modulation (a situation with the average dispersion slowly varying along the propagation distance is known in optics DM as well [17]). Since we are interested in the case of a sign-changing local dispersion, it is usually assumed that $\left|D_{1}\right| \leq S / 2$. In fact, without loss of generality, one may set $S=T=1$. Indeed, it is straightforward to see that Eq. (3) is mapped into itself, but with $S=T=1$, by transformation to new variables,

$$
\widetilde{u} \equiv \frac{u T}{(S T)^{1 / 3}}, \quad \widetilde{\chi} \equiv \frac{\chi}{T}, \quad \widetilde{\theta} \equiv \frac{\theta}{(S T)^{1 / 3}}, \quad \widetilde{D}(\tau) \equiv D_{0}(\tau)+S^{-1} D_{1}(T \tau) .
$$

In section 2, we develop an analytical approximation for solitary-wave solutions to Eq. (3) in the weakly nonlinear case, when the model can be reduced to a relatively simple integral equation. Results of direct simulations of the full model are displayed in section 3, demonstrating the existence of both stable and unstable solitons. In section 4, we briefly consider effects of the fifth-order dispersion, and the paper is concluded by section 5 . The appendix gives a short account of the derivation of Eq. (3), including the fifth-order-dispersion term, in a particular hydrodynamic problem.

\section{Dispersion-dominated waves}

Following the approach of Ref. [9], where the fiber-optic DM model was transformed into an integral equation, we consider Eq. (3) with the modulated dispersion (6) taken in the form

$$
D(\chi)=D_{0}(\chi)+\epsilon D_{1}(\epsilon \chi),
$$

where $\epsilon$ is a small parameter, $D_{0}(\chi)$ is a periodic function with period 1 , amplitude 1 and zero mean value and $\epsilon D_{1}(\epsilon \chi)$ is the slowly-varying small local average dispersion. We also assume that the initial value supplementing Eq. (3) is small, i.e. $f(\theta) \equiv u(\theta, \chi=0)=O(\epsilon)$. It is then natural to introduce a slow timescale $\tau \equiv \epsilon \chi$, redefine the initial value, so that

$$
f(\theta) \equiv \epsilon g(\theta),
$$

and look for a solution in the form

$$
u(\theta, \tau)=\epsilon u^{(0)}(\theta, \tau)+\epsilon^{2} u^{(1)}(\theta, \tau)+\cdots .
$$

Defining the "accumulated dispersion"

$$
W(\chi) \equiv \int_{0}^{\chi} D_{0}(\chi) d \chi
$$

the zeroth-order solution to Eq. (3) can be found in the form

$$
u^{(0)}=\mathcal{F}^{-1}\left\{\exp \left[i k^{3} W(\chi)\right] \mathcal{F}\{A(\theta, \tau)\}\right\},
$$

$\mathcal{F}$ and $\mathcal{F}^{-1}$ represent the Fourier transform with respect to $\chi$ and its inverse. From the initial condition (9), it follows that $A(\theta, 0)=g(\theta)$ in the solution (12). Because the function $D_{0}$ is periodic with zero mean, the functions $W(\chi)$ and $u^{(0)}$ given by Eqs. (11) and (12) are also periodic. 
At first order in $\epsilon$, Eq. (3) is reduced to

$$
u_{\chi}^{(1)}+D_{0} u_{\theta \theta \theta}^{(1)}=-\left[u_{\tau}^{(0)}+6 u^{(0)} u_{\theta}^{(0)}+D_{1}(\tau) u_{\theta \theta \theta}^{(0)}\right]
$$

with $u^{(1)}(\theta, 0, \tau)=0$. Defining

$$
h(k, \chi, \tau) \equiv \mathcal{F}\left\{u_{\tau}^{(0)}+6 u^{(0)} u_{\theta}^{(0)}+D_{1}(\tau) u_{\theta \theta \theta}^{(0)}\right\},
$$

Eq. (13) with the zero initial value has a solution

$$
u^{(1)}=-\mathcal{F}^{-1}\left\{\exp \left(i k^{3} W\right) \int_{0}^{\chi} \exp \left[-i k^{3} W(\sigma)\right] h(k, \sigma, \tau) d \sigma\right\} .
$$

To avoid the growth of secularities in the first-order solution (15), we must impose a condition

$$
\int_{0}^{1} \exp \left(-i k^{3} W(\chi)\right) h(k, \chi, \tau) d \chi=0,
$$

which can be written in terms of the Fourier transform, $\hat{A}(k)$, of the function $A(\theta)$ introduced in Eq. (12) as

$$
\int_{0}^{1}\left\{\hat{A}_{\tau}-i D_{0} k^{3} \hat{A}+3 i k \int_{-\infty}^{\infty} \hat{A}(\kappa) \hat{A}(k-\kappa) \exp [3 i k \kappa(\kappa-k) W(\chi)] d \kappa\right\} d \chi=0 .
$$

If we introduce a function

$$
V(\kappa) \equiv \int_{0}^{1} \exp [i \kappa W(\chi)]
$$

then it follows from Eq. (17) that the amplitude $A(\theta, \tau)$ satisfies the evolution equation:

$$
A_{\tau}+3 \mathcal{F}^{-1}\left\{i k \int_{-\infty}^{\infty} V(3 k \kappa(\kappa-k)) \hat{A}(\kappa) \hat{A}(k-\kappa) d \kappa\right\}+D_{0}(\tau) A_{\theta \theta \theta}=0,
$$

with the initial condition that $A(\theta, 0)=g(\theta)$.

The kernel (18) of the integro-differential equation (19) can be easily calculated in the case of the piecewise-constant dispersion, which is similar to the standard DM scheme in fiber-optic communications $[4,5]$,

$$
D_{0}(\chi)= \begin{cases}-1 / 2, & 1 / 4<\chi<3 / 4, \\ +1 / 2, & 3 / 4<\chi<5 / 4,\end{cases}
$$

which is repeated with period 1 . In this case, the result is

$$
V(\kappa)=(8 / \kappa) \sin (\kappa / 8) \text {. }
$$

For the sinusoidal modulation of the dispersion, with

$$
D_{0}(\chi)=(1 / 2) \cos (2 \pi \chi),
$$


the kernel can be found in the form

$$
V(\kappa)=\sum_{n=0}^{\infty} \frac{(-1)^{n}}{(n !)^{2}}\left(\frac{\kappa}{4}\right)^{2 n}
$$

In general, $V(\kappa) \rightarrow 1+O\left(\kappa^{2}\right)$ as $\kappa \rightarrow 0$, while for $\kappa \gg 1$ the integral can be evaluated using the stationary-phase approximation. The main contribution then comes from a vicinity of the point where $W^{\prime}(\chi) \equiv D_{0}(\chi)=0$. Assume that $D_{0}(\chi)$ has two zeroes, $\chi_{1}$ and $\chi_{2}$, which are symmetric, such that $\chi_{2}=1-\chi_{1}$ and $D_{0}^{\prime}\left(\chi_{1}\right)=-D_{0}^{\prime}\left(\chi_{2}\right)<0$ and $W\left(\chi_{1}\right)=-W\left(\chi_{2}\right)$. Then, the method of stationary-phase yields

$$
V(\kappa) \approx \sqrt{\frac{8 \pi}{\kappa D_{0}^{\prime}\left(\chi_{2}\right)}} \cos \left(\kappa W\left(\chi_{2}\right)+\frac{\pi}{4}\right) .
$$

Hereafter, we focus on steady-state solitary-wave solutions to Eqs. (19), following the approach of Ref. [9]. To this end, we assume that the average dispersion $D_{1}$ is a positive constant, and look for solutions in the form $A=$ $A(\theta-S \tau)$ with a constant velocity $S$. Anticipating that $S \geq 0$, we set $S \equiv \lambda^{2}$, so that the Fourier transform $\hat{A}(k)$ must satisfy an equation following from Eq. (19),

$$
\left(\lambda^{2}+D_{1} k^{2}\right) \hat{A}=3 \int_{-\infty}^{\infty} V(3 k \kappa(\kappa-k)) \hat{A}(\kappa) \hat{A}(k-\kappa) d \kappa
$$

By means of the definition $\hat{A}(k) \equiv \lambda^{2} \hat{B}(k)$, one can combine two free parameters $D_{1}$ and $\lambda^{2}$ into a single one, $D_{1} / \lambda^{2}$. Then, given the number $N$ of Fourier modes, the following iterative scheme is used to solve the discrete truncated version of Eq. (25):

$$
\begin{aligned}
& \hat{B}_{k}^{(n)}=\frac{3}{\lambda^{2}+D_{1} k^{2}} \sum_{l=-N / 2}^{N / 2} V(3 k l(l-k)) \hat{A}_{l}^{(n)} \hat{A}_{k-l}^{(n)}, \\
& \hat{A}_{k}^{(n+1)}=\frac{\hat{P}\left\{\hat{A}^{(n)}\right\}}{\hat{P}\left\{\hat{A}^{(n)}\right\}} \hat{B}_{k}^{(n)}
\end{aligned}
$$

Here, the superscript is the iteration number, while the integer argument $k$ is written as the subscript attached to the amplitudes $\hat{B}^{(n)}$ and $\hat{A}^{(n)}$, and

$$
\hat{P}\{\hat{A}\} \equiv \sum_{j=0}^{N-1}\left(\hat{A}_{j}\right)^{2}
$$

is the momentum (see Eq. (5)) of the discrete field $\hat{A}_{j}$.

In Figs. 1 and 2, localized solutions obtained by means of the numerically implemented iterative scheme (26), (27) are shown as $D_{1}$ is varied for the two forms (20) and (22) of the dispersion modulation. Note that localized solutions with positive extrema appear to be only possible for $D_{1} \geq 0$. By symmetry, localized solutions with negative extrema and negative velocity are then only possible for $D_{1} \leq 0$. As can be seen, a significant difference between the waves for each dispersion-modulation form is observed only near $D_{1}=0$. For 


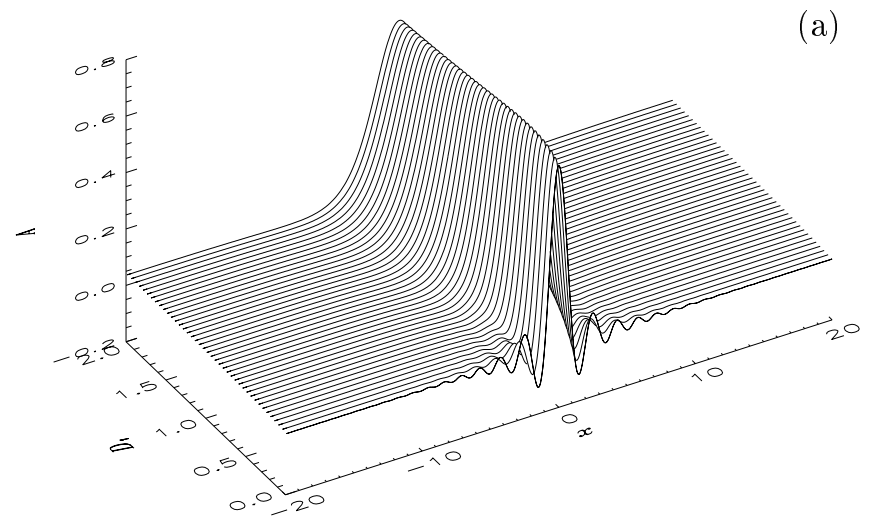

(b)

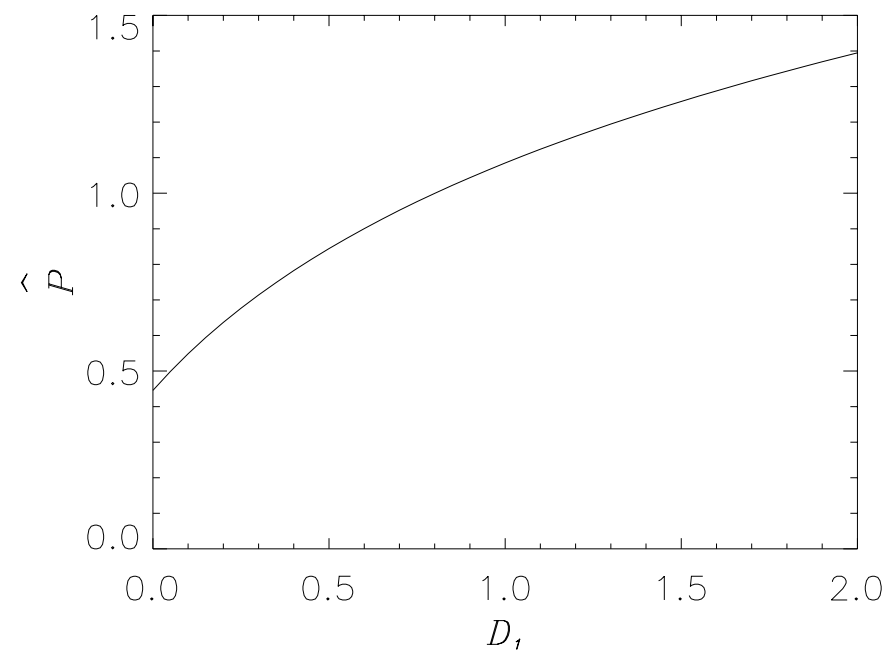

Figure 1: Localized solutions of Eq. (25), obtained for the piecewise-constant dispersion modulation (21) and $\lambda^{2}=1$. In (a) the shape of the pulse, and in (b) the variation of the momentum $\hat{P}$ are shown as $D_{1}$ is varied. 


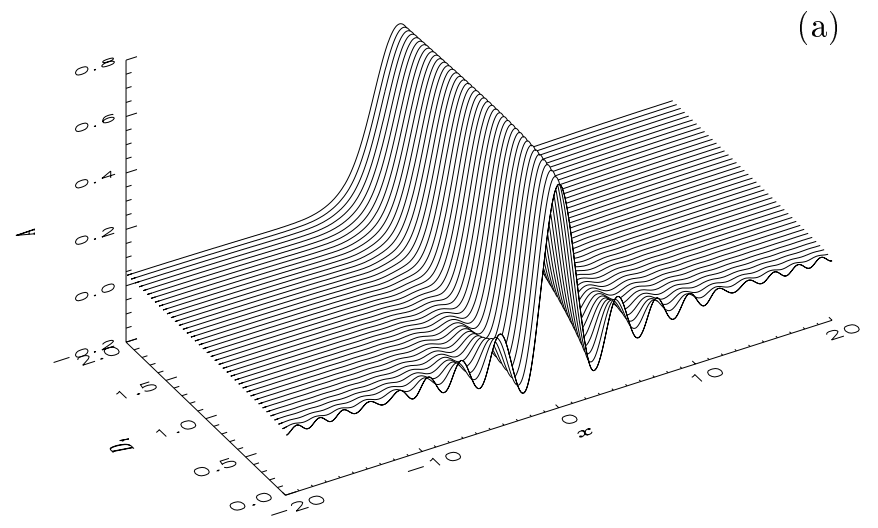

(b)

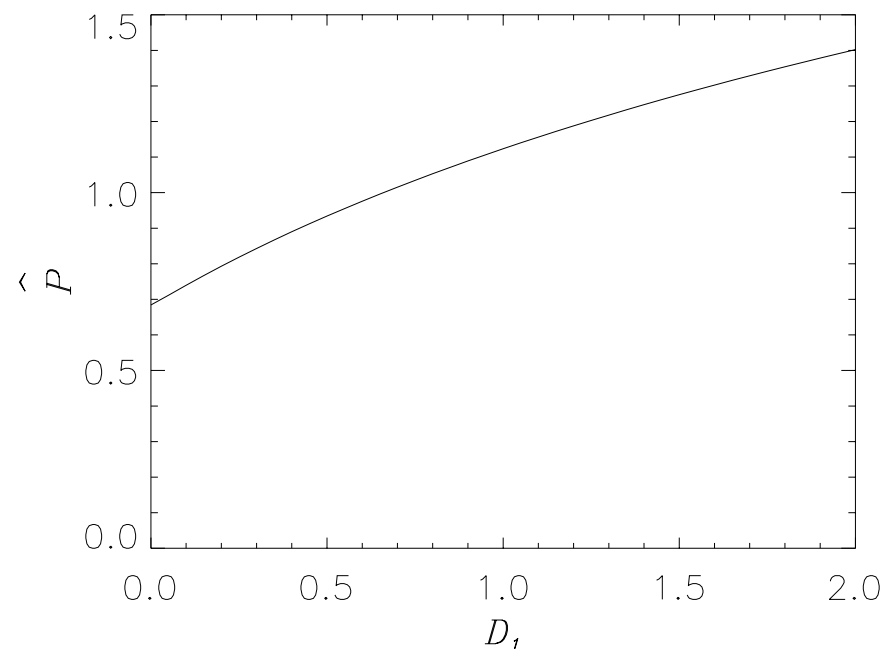

Figure 2: The same as in Fig. 1, but for the harmonic dispersion modulation (23). 


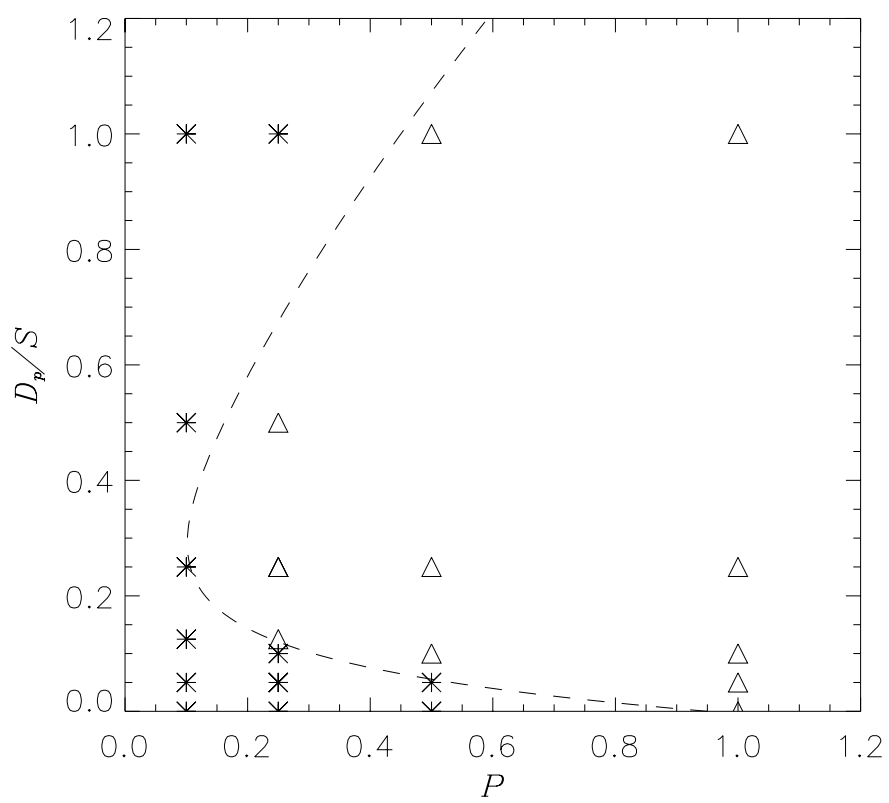

Figure 3: The stability boundary for soliton solutions to Eq. (3) for the piecewise dispersion modulation (20). Stable and unstable solutions are denoted by asterisks and triangles respectively.

larger $D_{1}$, the mean dispersion dominates, and the waves for both types of the modulation approach the classical $\mathrm{sech}^{2}$ soliton of the constant-coefficient KdV equation. For clarity, only the domain $-20 \leq x \leq 20$ is shown in these figures; in larger domains, the oscillations apparent in Fig. 2 near $D_{1}=0$ decay to zero.

\section{$3 \quad$ Numerical results}

A crucially important issue is stability of the stationary solitary waves obtained in the previous section by means of the perturbation expansion. This could be addressed by extending the perturbation expansion to the next order, but this approach proves unwieldy. A simpler approach is to use solutions of Eq. (25) as initial conditions for the underlying equation (3), and then to follow the evolution in direct simulations. To solve Eq. (3) numerically, we used a standard method combining pseudo-spectral techniques in $\theta$ and third-order Runge-Kutta integration in $\chi$.

In this section, we consider only the case when average dispersion $D_{1}$ is constant. Further, the results presented here are obtained for a slightly smoothed version of the piecewise-constant dispersion modulation (20). Results for other forms of the dispersion modulation, including the sinusoidal form (22), are very similar. Quantitative results, such as the exact position of the stability boundary in Fig. 3, see below, may be affected by the choice of the modulation form. 
The transformation (7) demonstrates that, given a particular form of the dispersion modulation, the ratio $D_{1} / S$ and momentum $P$ uniquely define a dispersion-dominated solution. Thus, when investigating the stability of dispersiondominated solutions of Eq. (3), we only have the two free parameters $D_{1} / S$ and $P$. For the modulation form (20), numerically found stability boundaries of the dispersion-dominated solutions are shown in figure 3.

In classifying the solutions as stable or unstable, we have used a simple criterion that, for unstable solutions, in some region the Poincaré map of the solution's amplitude (maximum value) will undergo sustained mean algebraic decay in the variable $\chi$. Since we solve Eq. (3) with periodic boundary conditions in $\theta$, at a late asymptotic stage of the evolution, the decay of the soliton will be followed by establishment of a nearly uniform state, in which maximum and minimum values of the field are approximately equal and constant in $\chi$. Conversely, for stable soliton solutions, we expect that, asymptotically, the absolute mean of the Poincaré map of the maximum value of the field will be much larger than its minimum-value counterpart. The stability boundary depends on a number of factors, in particular, the computational domain size $L$ and grid size $\Delta \theta$. However, we have taken care to keep $L$ sufficiently large and $\Delta \theta$ sufficiently small to minimize their effect. The boundary also depends on the final propagation distance, $\chi_{f}$. All the solutions shown in figure 3 used $\chi_{f}=400$, which was found to be sufficiently large to make it possible to conclude whether a stable soliton was established, or the solution underwent decay.

Figure 3 clearly shows that for this problem there are two distinct regions of stable solitary-wave solutions. The stability boundary corresponds to some curve $P_{b}=P\left(D_{1} / S\right)$, with the minimum value of $P_{b}$ occurring at a critical value $R_{c}$ of $D_{1} / S$, where for the piecewise-constant modulation $R_{c} \approx 0.25$. Then, the stable region in $0 \leq D_{1} / S<R_{c}$ will be referred to as "dispersiondominated", while that in $D_{1} / S>R_{c}$ will be referred to as the "classical" region. The numerical results suggest that the dispersion-dominated region decreases exponentially with the increase of $D_{1} / S$, while the classical region increases algebraically with the increase of $D_{1} / S$. In the dispersion-dominated region for $D_{1} \equiv 0$, it is apparent that localized solutions of Eq. (3) are only possible up to a finite value of the momentum. For the piecewise-constant dispersion modulation, this critical value was found to be $P \approx 0.95$. Thus, for the variable-dispersion $\mathrm{KdV}$ equation both nonlinearity and mean dispersion act to destabilize the dispersion-dominated solitons. The growth of the classical region as $D_{1} / S$ is increased is easily understood. In this region, solitary-wave solutions are due to balance between nonlinearity and mean dispersion, the effect of variable dispersion is then simply to modulate the form of the wave. In this region, solutions can be investigated using perturbation theory for the classical $\mathrm{sech}^{2}$ solitons [16], or using methods similar to the guiding-center technique, which were developed for solitons in optical fibers [18].

In Figs. 4, 5 and 6 examples of solutions corresponding to particular points in Fig. 3 are shown. These three soliton solutions are, respectively, stable, unstable, and stable again. The dispersion-dominated solution in Fig. 4 demonstrates that some momentum is lost from the localized wave to higher mode oscillatory waves. The subsequent interaction between the oscillatory waves and the solitary wave causes relatively large variability in the Poincare map of the maximum value of the field; however, both the maximum and minimum values eventually set down to mean values not significantly different from their 
(a)

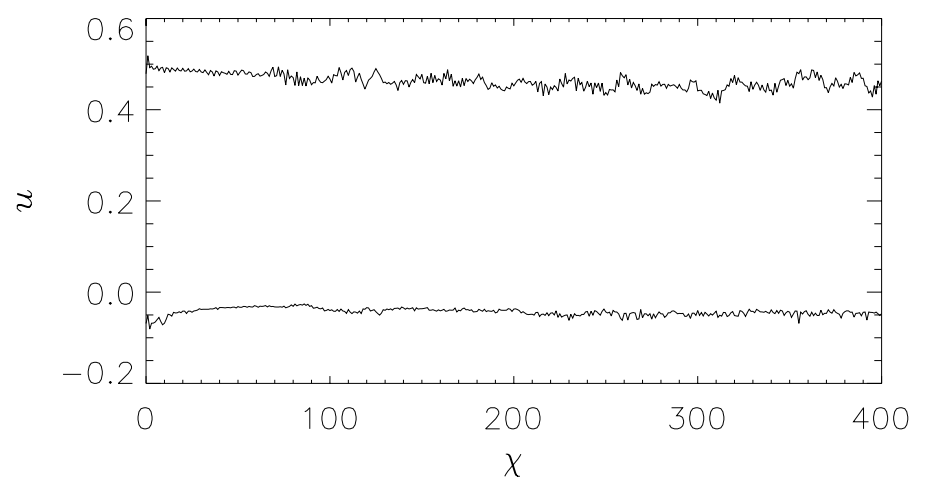

(b)

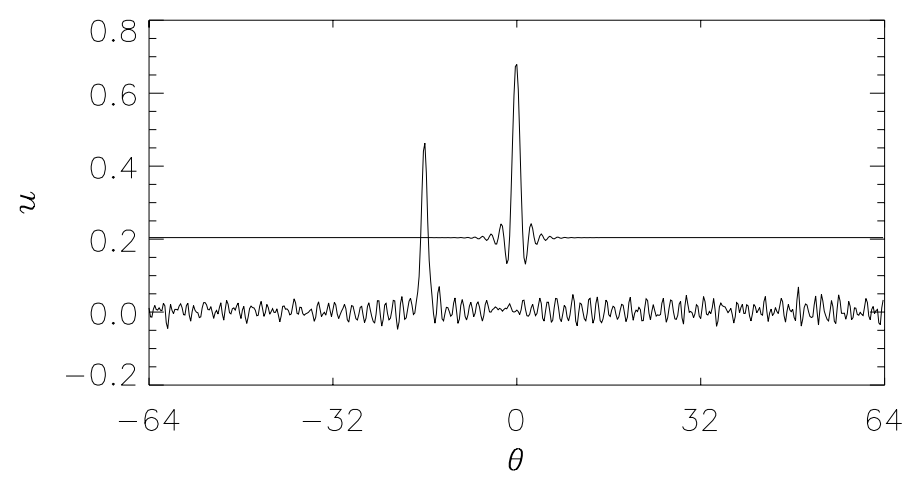

Figure 4: A stable dispersion-dominated solution, corresponding to the point $P=0.25, D_{1} / S=0.05$ from Fig. 3. (a) The Poincare map of the maximum and minimum values of the field, i.e., the maximum and minimum values shown at integer values of the propagation distance (evolutional variable) $\chi$. These plots clearly show that stable nearly stationary solitons have been found. (b) The form of the soliton at $\chi=0$ (displaced by 0.2 ) and 400 .

initial values. The decay of a localized wave is apparent in Fig. 5, where the mean of both the maximum and minimum values of the field can be seen to decrease. Eventually, at large enough propagation distance they would be expected to become approximately equal in the absolute values and opposite in the sign. At this stage, the mass and momentum contained initially in the localized wave would be completely transferred to the oscillatory waves apparent in Fig. 5(b). For large values of the average dispersion, as is shown in Fig. 6, which is an example of a soliton belonging to the classical region, it is apparent that the solitary wave solutions are now very similar to the $\mathrm{sech}^{2}$ solitons of the constant-dispersion $\mathrm{KdV}$ equation. Here, only a small amount of the momentum from the initial wave is lost to the oscillatory waves. Because of the reduced amplitude of the oscillatory wave, the oscillations in the Poincare map of the maximum field value are now much slower than in Fig. 4. 
(a)

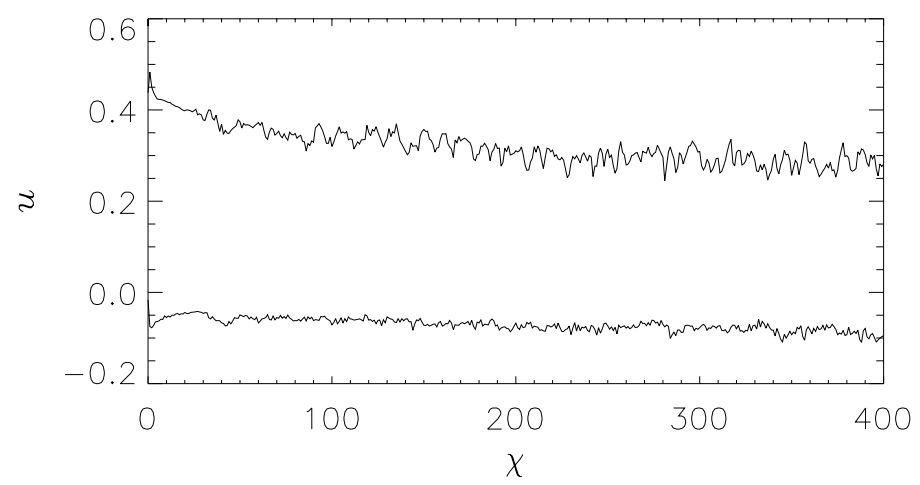

(b)

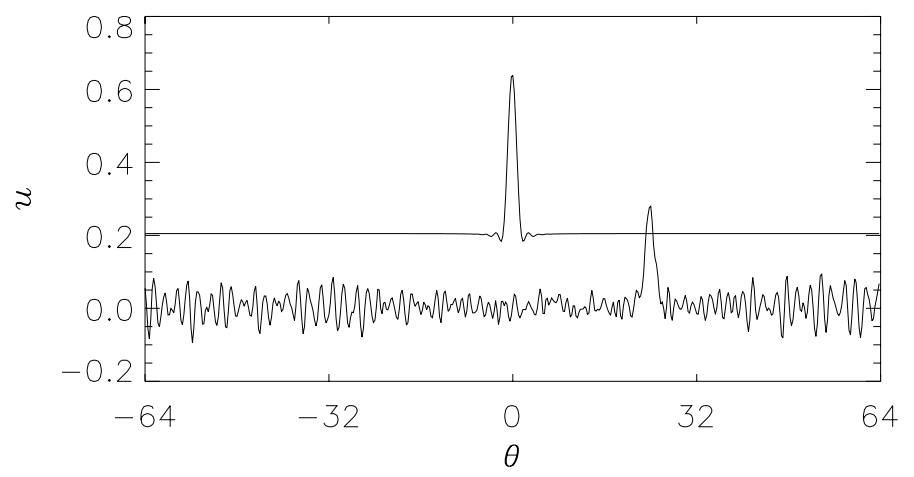

Figure 5: The same as in Fig. 4 for $P=0.25$ and $D_{1} / S=0.25$. In this case, the soliton is unstable. 
(a)

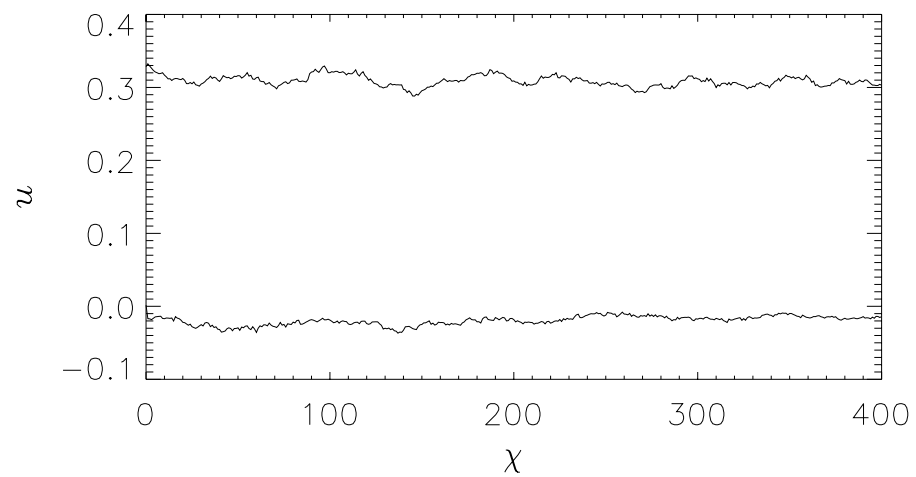

(b)

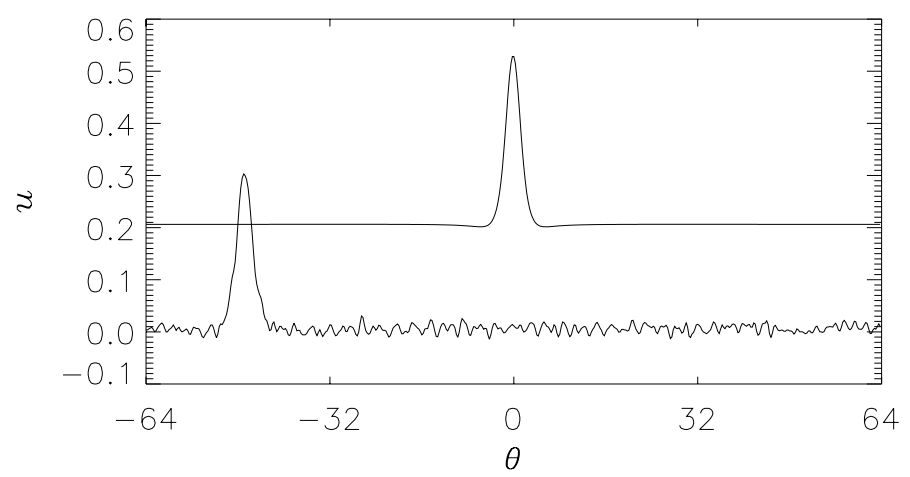

Figure 6: The same as in Fig. 4 for a stable soliton solution with $P=0.25$ and $D_{1}=S$ in the classical region. 


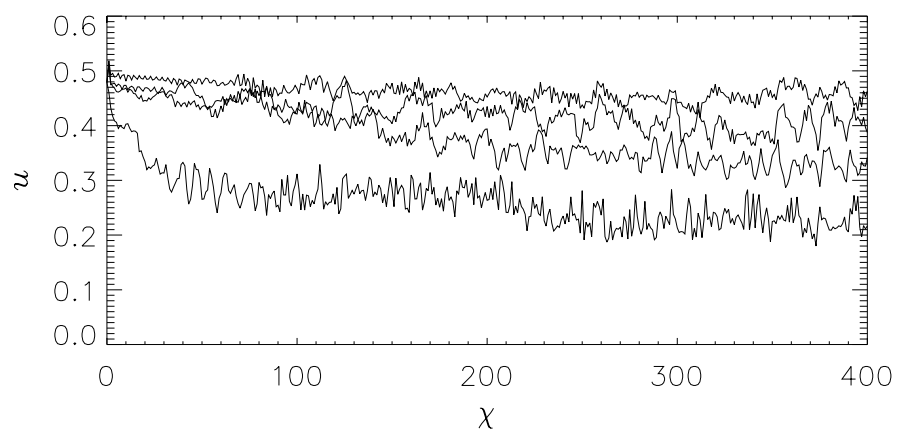

Figure 7: The Poincaré map of the maximum values of the field, as obtained from the numerical solution of Eq. (29) for $\delta^{2}=0,10^{-3}, 5 \times 10^{-3}$, and $10^{-2}$. Increasing $\delta^{2}$ corresponds to quicker decay of the amplitude. This shows the effect of the fifth-order dispersion on the otherwise stable dispersion-dominated solution from figure 4 .

\section{Effects of fifth-order dispersion}

As it was mentioned above, the fifth-order dispersion should, generally speaking, be added to the model of the DM type with a small average dispersion (similarly, the third-order dispersion should be included into the optical DM models [19]). The main effect on the solitons will be to cause them to disperse. In particular, one may expect that, for small values of the average dispersion, the addition of fifth-order dispersion to Eq. (3) will eventually cause stable solutions to become unstable. It is expected that this effect is most important when the third-order dispersion undergoes a change in sign, i.e. for $D_{1}<S / 2$ in Fig. 3. For larger values of $D_{1}$, it is expected that the main effect of the fifth-order dispersion will be to enhance the generation of oscillatory waves [20]. Therefore, we concentrate here on the effect of fifth-order dispersion on the dispersion-dominated solutions.

With the addition of fifth-order dispersion, Eq. (3) becomes

$$
u_{\chi}+6 u u_{\theta}+D(\chi) u_{\theta \theta \theta}+\delta^{2} u_{\theta \theta \theta \theta \theta}=0,
$$

where, as shown for the case of gravity-capillary waves in the Appendix, one can assume that the coefficient $\delta^{2}$ is constant, in contrast to the strongly modulated third-order-dispersion coefficient. In Fig. 7, the effect of varying this parameter on the stable solution from Fig. 4 is shown. First, the case of $\delta^{2}=10^{-4}$ was investigated, but it is not shown in Fig. 7, as it turns out to be practically identical to $\delta^{2}=0$. Thus, for sufficiently small $\delta^{2}$, stable solitary waves are established as well as in the case $\delta^{2}=0$. However, as $\delta^{2}$ is increased, the solitons disperse, leaving oscillatory-wave radiation. Other simulations in the dispersion-dominated region confirm that the critical (maximum) value of $\delta^{2}$, at which the solitary waves are able to form, decreases as both $D_{1} / S$ and $P$ increase, so that on the stability boundary from Fig. 3 the critical value is $\delta^{2}=0$. 


\section{Conclusions}

In this work we have introduced a $\mathrm{KdV}$ model with a periodically varying dispersion coefficient that takes both positive and negative values. Using a perturbative expansion based on integral equations and direct numerical simulations, we have shown that for the case when the dispersion undergoes a periodic change in sign, stable solitary-wave solutions are possible in a region defined by the average dispersion and the initial momentum of the system. Further, it has been shown that sufficiently weak fifth-order dispersion, which should be considered when the third-order dispersion attains a value of zero, does not destroy these solitary waves structures.

It should be noted that only one analytical approach to investigate the possibility of solitary-wave solutions has been presented in detail in this work. Other approaches may predict the existence of a larger region for solitary-wave solutions than that found numerically and shown in Fig. 3. We have also tried two additional approaches for considering soliton solutions. The first of these is the balance-equation method. Equation (3) can be derived from the Hamiltonian

$$
H=\int_{-\infty}^{+\infty}\left[-\frac{1}{2} D(\chi) u_{\theta}^{2}+u^{3}\right] d \theta,
$$

which, unlike the mass and momentum given by Eq. (5), is not conserved when the dispersion coefficient is variable. Instead, it evolves with $\chi$ according to an immediate corollary of (3),

$$
\frac{d H}{d \chi}+\frac{1}{2} \frac{d D}{d \chi} \int_{-\infty}^{+\infty} u_{\theta}^{2} d \theta=0 .
$$

The balance-equation approach then demands a choice of a slowly varying ansatz for the form of the solitary wave. Using the conservation equations for the mass and momentum, and the evolution equation (31) for the Hamiltonian, evolution equations for parameters of the ansatz can be derived. The second approach is a numerical averaging procedure, involving successive Poincaré maps from direct numerical simulations of Eq. (3) in an attempt to iterate to a stable soliton solution. However, neither of these methods have proved successful for the variable-dispersion $\mathrm{KdV}$ equation.

The reason for solitary-wave solutions being less forthcoming from the variabledispersion $\mathrm{KdV}$ equation than for the variable-dispersion NLS equation may have to do with the zero-dispersion limit of these two equations. For the $\mathrm{KdV}$ equation, it is a singular limit with wavebreaking occurring in finite time, whereas for the NLS equation in the zero-dispersion limit, nothing but pure phase modulation takes place. Thus, when nonlinearity dominates over the variable dispersion in the $\mathrm{KdV}$ equation, as in the limit $D_{1} / S=0$ and $P \gg 1$ in Fig. 3 above, one would not expect that stable solutions are possible, as the evolution generated by the nonlinearity is much faster than that under the action of the variable dispersion. Nonetheless, combining the results presented here with the well-known ones for the dispersion-managed solitons in the NLS models of long fiber-optic links consisting of alternating segments with anomalous and normal dispersion, we conjecture that stable pulsating solitary waves may be possible in a vast class of nonlinear wave equations subject to strong periodic modulation of the dispersion coefficient. 


\section{Acknowledgment}

B.A.M. appreciates the hospitality of Loughborough University (UK) and Monash University (Clayton, Australia).

\section{A Long, weakly-nonlinear gravity-capillary waves over slowly varying topography near the crit- ical Bond number}

Consider the propagation of long, weakly-nonlinear gravity-capillary waves in water of depth $h$ with horizontal lengthscale $L=h / \delta$ and amplitude $a=\epsilon h$, such that $\delta, \epsilon \ll 1$. Let $g$ be the gravity acceleration, $\rho$ the density of the water, and $\sigma$ the coefficient of surface tension. Then the evolution of the free-surface displacement, $\epsilon \eta$, as a function of the time, $t / \delta$, and horizontal coordinate, $x / \delta$, for one-dimensional waves propagating to the right, is governed by the extended $\mathrm{KdV}$ equation [20]

$$
\frac{1}{c_{0}} \frac{\partial \eta}{\partial t}+\left(1+\epsilon \frac{3 \eta}{2 h}\right) \frac{\partial \eta}{\partial x}+\delta^{2} \alpha h^{2} \frac{\partial^{3} \eta}{\partial x^{3}}+\delta^{4} \beta h^{4} \frac{\partial^{5} \eta}{\partial x^{5}}=O\left(\epsilon^{2}, \epsilon \delta^{2}, \delta^{6}\right),
$$

where

$$
\alpha=\frac{1}{2}\left(\frac{1}{3}-B\right), \quad \beta=\frac{1}{2}\left(\frac{2}{15}-\frac{B}{3}-\alpha^{2}\right), \quad c_{0}=(g h)^{1 / 2} .
$$

and $B=\sigma / \rho g h^{2}$ is the Bond number. Now assume in addition that the depth is a slowly varying function of $x$, such that $h=h(\epsilon x)$, and introduce the new variables

$$
\chi=\epsilon x, \quad \theta=g^{\frac{1}{2}}\left(t-\int \frac{d x}{c}\right),
$$

Further, we put $A \equiv \eta h^{\frac{1}{4}}$ and note that in a slowly varying environment the wave action flux density $A^{2}$ is conserved $[3,16]$. Hence, the corrected form of Eq. (32) is

$$
\epsilon \frac{\partial A}{\partial \chi}-\epsilon \frac{3 A}{2 h^{\frac{7}{4}}} \frac{\partial A}{\partial \theta}-\delta^{2} \alpha h^{\frac{1}{2}} \frac{\partial^{3} A}{\partial \theta^{3}}-\delta^{4} \beta h^{\frac{3}{2}} \frac{\partial^{5} A}{\partial x^{5}}=O\left(\epsilon^{2}, \epsilon \delta^{2}, \delta^{6}\right) .
$$

Typically $\alpha$ is $O(1)$; then, let $\epsilon=\delta^{2}$ and the propagation of waves is governed by the variable-coefficient $\mathrm{KdV}$ equation

$$
\frac{\partial A}{\partial \chi}-\frac{3 A}{2 h^{\frac{7}{4}}} \frac{\partial A}{\partial \theta}-\alpha h^{\frac{1}{2}} \frac{\partial^{3} A}{\partial \theta^{3}}=0 .
$$

However, $B=1 / 3$ is a singular limit of this equation; in this limit we set $\alpha=\delta^{2} \gamma$ and $\epsilon=\delta^{4}$, then Eq. (35) becomes

$$
\frac{\partial A}{\partial \chi}-\frac{3 A}{2 h^{\frac{7}{4}}} \frac{\partial A}{\partial \theta}-\gamma h^{\frac{1}{2}} \frac{\partial^{3} A}{\partial \theta^{3}}-\beta h^{\frac{3}{2}} \frac{\partial^{5} A}{\partial \theta^{5}}=0,
$$


where now $\beta=1 / 90$. Thus, if the topography and Bond number are such that the coefficient $\alpha$ is close to zero, and can change its sign, then a uniformly valid model equation including the fifth-order dispersion is

$$
\frac{\partial A}{\partial \chi}-\frac{3 A}{2 h^{\frac{7}{4}}} \frac{\partial A}{\partial \theta}-\alpha h^{\frac{1}{2}} \frac{\partial^{3} A}{\partial \theta^{3}}-\frac{\delta^{2} h^{\frac{3}{2}}}{90} \frac{\partial^{5} A}{\partial \theta^{5}}=0 .
$$

\section{References}

[1] G.P. Agrawal. Nonlinear Fiber Optics (Academic Press: Boston, 1995).

[2] S.A. Maslowe and L.G. Redekopp, J. Fluid Mech. 101, 321 (1980). K.-K. Tung, D.R.S. Ko and J.J. Chang, Stud. Appl. Math. 65, 189 (1981). S.A. Maslowe and S.R. Clarke, Stud. Appl. Math. (submitted).

[3] R. Grimshaw, in Advances in Coastal and Ocean Engineering, ed. P.L.-F. Liu, World Scientific Publishing Company, Singapore, 3, 1 (1997).

[4] F.M. Knox, N.J. Doran, K.J. Blow and I. Bennion, Electron. Lett. 32, 54 (1996); I. Gabitov and S.K. Turitsyn, Opt. Lett. 21, 327 (1996).

[5] New Trends in Optical Soliton Transmission Systems, ed. by A. Hasegawa (Kluwer Academic Publishers: Dordrecht/Boston/London, 1998).

[6] A. Berntson, N.J. Doran, W. Forysiak, and J.H.B. Nijhof, Opt. Lett. 23, 900 (1998).

[7] A. Berntson, D. Anderson, M. Lisak, M.L. Quiroga-Teixeiro and M. Karlsson, Opt. Commun. 130, 153-162 (1996); I. Gabitov, E.G. Shapiro and S.K. Turitsyn, Opt. Commun. 134, 317 (1997); B.A. Malomed, Opt. Commun. 136, 313 (1997); T.-S. Yang and W.L. Kath, Opt. Lett. 22, 985 (1997); M. Matsumoto, Opt. Lett. 22, 1238 (1997); S.K. Turitsyn et al., Opt. Commun. 151, 117 (1998).

[8] T.I. Lakoba, J. Yang, D.K. Kaup, and B.A. Malomed, Opt. Commun. 149, 366 (1998).

[9] M.J. Ablowitz and G. Biondini, Opt. Lett. 23, 1668 (1998).

[10] J.H.B. Nijhof, N.J. Doran, W. Forysiak and F.M. Knox, Electron. Lett. 33, 1726 (1997); S.K. Turitsyn and E.G. Shapiro, Opt. Lett. 23, 682 (1998); J.N. Kutz and S.G. Evangelides, Opt. Lett. 23, 685 (1998).

[11] B.A. Malomed, D.F. Parker, and N.F. Smyth, Phys. Rev. E 48, 1418 (1993); R. Grimshaw, J. He and B.A. Malomed, Physica Scripta 53, 385 (1996); F.Kh. Abdullaev and J.C. Caputo, Phys. Rev. E 58, 6637 (1998).

[12] L. Torner, IEEE Photon. Techn. Lett. 11, 1268 (1999).

[13] R. Driben and B.A. Malomed, Opt. Commun. 185, 439 (2000).

[14] L. Bergé, V.K. Mezentsev, J. Juul Rasmussen, P.L. Christiansen, and Yu.B. Gaididei, Opt. Lett. 25, 1037 (2000).

[15] B.V. Gisin, A. Kaplan, and B.A. Malomed, to be published. 
[16] D.J. Kaup and A.C. Newell, Proc. Roy. Soc. Lond. A 361, 413 (1978); Yu.S. Kivshar and B.A. Malomed, Rev. Mod. Phys. 61, 763 (1989).

[17] B.A. Malomed, Opt. Commun. 147, 157 (1998); M. Zitelli, B. Malomed, F. Matera, and M. Settembre, Opt. Commun. 154, 273 (1998).

[18] V. Cautaerts, A. Maruta and Y. Kodama, Chaos 10, 515 (2000).

[19] D. Frantzeskakis et al., Pure Appl. Opt. 7, L57 (1998).

[20] J.K. Hunter and J. Scheurle, Physica D 32, 253 (1988); R. Grimshaw and N. Joshi, SIAM J. Appl. Math 55, 124 (1995). 